\title{
A Kantorovich-Stancu Type Generalization of Szasz Operators including Brenke Type Polynomials
}

\author{
Rabia Aktaş, ${ }^{1}$ Bayram Çekim, ${ }^{2}$ and Fatma Taşdelen ${ }^{1}$ \\ ${ }^{1}$ Department of Mathematics, Faculty of Science, Ankara University, Tandoğan, 06100 Ankara, Turkey \\ ${ }^{2}$ Department of Mathematics, Faculty of Science, Gazi University, Beşevler, 06500 Ankara, Turkey
}

Correspondence should be addressed to Rabia Aktaş; raktas@science.ankara.edu.tr

Received 11 September 2013; Accepted 18 November 2013

Academic Editor: Anna Kaminska

Copyright (c) 2013 Rabia Aktaş et al. This is an open access article distributed under the Creative Commons Attribution License, which permits unrestricted use, distribution, and reproduction in any medium, provided the original work is properly cited.

We introduce a Kantorovich-Stancu type modification of a generalization of Szasz operators defined by means of the Brenke type polynomials and obtain approximation properties of these operators. Also, we give a Voronovskaya type theorem for KantorovichStancu type operators including Gould-Hopper polynomials.

\section{Introduction}

For each positive $n$ and $f \in C_{B}([0, \infty))$ or $C([0, \infty)) \cap E$, the Szasz-Mirakyan operators defined by

$$
S_{n}(f ; x):=e^{-n x} \sum_{k=0}^{\infty} \frac{(n x)^{k}}{k !} f\left(\frac{k}{n}\right)
$$

have an important role in the approximation theory [1]. Their Korovkin type approximation properties and rates of convergence have been investigated by many researchers. Recently, there is a growing interest in defining linear positive operators via special functions (see [2-13]). In particular, many authors have studied various generalizations of Szasz operators via special functions. In [14], Jakimovski and Leviatan constructed a generalization of Szasz operators by means of the Appell polynomials. Then, Ismail [15] presented another generalization of Szasz operators by means of Sheffer polynomials, which involves the operators (1) defined by Jakimovski and Leviatan in [14]. In [11],Varma et al. considered the following generalization of Szasz operators by means of the Brenke type polynomials, which are motivated by the operators defined by Jakimovski and Leviatanand Ismail, for $x \geq 0$ and $n \in \mathbb{N}$ :

$$
L_{n}(f ; x):=\frac{1}{A(1) B(n x)} \sum_{k=0}^{\infty} p_{k}(n x) f\left(\frac{k}{n}\right)
$$

under the following assumptions:

$$
\begin{aligned}
& \text { (i) } A(1) \neq 0, \quad \frac{a_{k-r} b_{r}}{A(1)} \geq 0, \quad 0 \leq r \leq k, k=0,1,2, \ldots \text {, } \\
& \text { (ii) } B:[0, \infty) \longrightarrow(0, \infty), \\
& \text { (iii) (4) and (5) converge for }|t|<R \quad(R>1),
\end{aligned}
$$

where

$$
\begin{gathered}
A(t)=\sum_{r=0}^{\infty} a_{r} t^{r}, \quad a_{0} \neq 0, \\
B(t)=\sum_{r=0}^{\infty} b_{r} t^{r}, \quad b_{r} \neq 0 \quad(r \geq 0)
\end{gathered}
$$

are analytic functions and the Brenke type polynomials [16] have generating functions of the form

$$
A(t) B(x t)=\sum_{k=0}^{\infty} p_{k}(x) t^{k}
$$

where

$$
p_{k}(x)=\sum_{r=0}^{k} a_{k-r} b_{r} x^{r}, \quad k=0,1,2, \ldots
$$


The Kantorovich type of Szasz-Mirakyan operators is defined by [17]

$$
K_{n}(f ; x):=n e^{-n x} \sum_{k=0}^{\infty} \frac{(n x)^{k}}{k !} \int_{k / n}^{(k+1) / n} f(t) d t .
$$

The approximation properties of the Szasz-MirakyanKantorovich operators and their various iterates were studied by many authors in $[12,18-23]$.

Recently, in [8], the Kantorovich type of the operators given by (2) under the assumptions (3) has been defined as

$$
K_{n}(f ; x):=\frac{n}{A(1) B(n x)} \sum_{k=0}^{\infty} p_{k}(n x) \int_{k / n}^{(k+1) / n} f(t) d t,
$$

where $n \in \mathbb{N}, x \geq 0$ and $f \in C[0, \infty)$, and some of its properties have been investigated.

The purpose of this study is to introduce a KantorovichStancu type modification of the operators given by (8) and to examine the approximation properties of these operators. We also present a Kantorovich-Stancu type of the operators including Gould-Hopper polynomials and then we prove a Voronovskaya type theorem for these operators including Gould-Hopper polynomials.

\section{Construction of the Operators}

For each positive integer $n, x \geq 0$ and $f \in C_{B}([0, \infty))$, or $C([0, \infty)) \cap E$, let us consider the following operators:

$$
K_{n}^{(\alpha, \beta)}(f ; x):=\frac{n+\beta}{A(1) B(n x)} \sum_{k=0}^{\infty} p_{k}(n x) \int_{(k+\alpha) /(n+\beta)}^{(k+\alpha+1) /(n+\beta)} f(t) d t,
$$

where $\alpha$ and $\beta$ parameters satisfy the condition $0 \leq \alpha \leq \beta$. For the approximation properties of Stancu type operators, we refer to [24-27].

It is clear that for $\alpha=\beta=0, K_{n}^{(\alpha, \beta)}(f ; x)$ reduces to the operators defined by (8).

In the case of $B(t)=e^{t}$ and $A(t)=1$, with the help of (5) it follows that $p_{k}(x)=x^{k} / k$ !. So the operator $K_{n}^{(\alpha, \beta)}(f ; x)$ gives the Kantorovich-Stancu type of Szasz-Mirakyan operators as follows:

$$
K_{n}^{(\alpha, \beta)}(f ; x):=(n+\beta) e^{-n x} \sum_{k=0}^{\infty} \frac{(n x)^{k}}{k !} \int_{(k+\alpha) /(n+\beta)}^{(k+\alpha+1) /(n+\beta)} f(t) d t,
$$

where $\alpha$ and $\beta$ parameters satisfy the condition $0 \leq \alpha \leq \beta$.

In the case of $\alpha=\beta=0$, the operator (10) turns out to be the Szasz-Mirakyan-Kantorovich operators given by (7).

For $B(t)=e^{t}, K_{n}^{(\alpha, \beta)}(f ; x)$ gives the Kantorovich-Stancu type of the operators $P_{n}(f ; x)$ proposed by Jakimovski and Leviatan in [14].

Now, for the operators $K_{n}^{(\alpha, \beta)}$ given by (9), we give some results which are necessary to prove the main theorem.

Lemma 1. Kantorovich-Stancu type operators, defined by (9), are linear and positive.
Lemma 2. For each $x \in[0, \infty)$, the Kantorovich-Stancu type operators (9) have the following properties:

$$
\begin{aligned}
& K_{n}^{(\alpha, \beta)}(1 ; x)=1, \\
& K_{n}^{(\alpha, \beta)}(s ; x)=\frac{n}{n+\beta} \frac{B^{\prime}(n x)}{B(n x)} x+\frac{A^{\prime}(1)}{(n+\beta) A(1)}+\frac{2 \alpha+1}{2(n+\beta)}, \\
& K_{n}^{(\alpha, \beta)}\left(s^{2} ; x\right)=\left.\frac{(12)}{n+\beta}\right)^{2} \frac{B^{\prime \prime}(n x)}{B(n x)} x^{2} \\
&+\frac{n B^{\prime}(n x)\left[2 A^{\prime}(1)+(2 \alpha+2) A(1)\right]}{(n+\beta)^{2} A(1) B(n x)} x \\
&+\frac{1}{(n+\beta)^{2} A(1)}\left\{A^{\prime \prime}(1)+(2 \alpha+2) A^{\prime}(1)\right. \\
&\left.+\left(\alpha^{2}+\alpha+\frac{1}{3}\right) A(1)\right\} .
\end{aligned}
$$

Proof. From the generating function of the Brenke type polynomials given by (5), a few calculations reveal that

$$
\begin{gathered}
\sum_{k=0}^{\infty} p_{k}(n x)=A(1) B(n x), \\
\sum_{k=0}^{\infty} k p_{k}(n x)=A^{\prime}(1) B(n x)+n x A(1) B^{\prime}(n x) \\
\sum_{k=0}^{\infty} k^{2} p_{k}(n x)=n^{2} x^{2} A(1) B^{\prime \prime}(n x) \\
+n x B^{\prime}(n x)\left\{2 A^{\prime}(1)+A(1)\right\} \\
+B(n x)\left\{A^{\prime \prime}(1)+A^{\prime}(1)\right\} .
\end{gathered}
$$

By using these equalities, we obtain the assertions of the lemma by simple calculation.

Lemma 3. For each $x \in[0, \infty)$, one has

$$
\begin{aligned}
K_{n}^{(\alpha, \beta)}\left((s-x)^{2} ; x\right) & \\
= & \left\{\left(\frac{n}{n+\beta}\right)^{2} \frac{B^{\prime \prime}(n x)}{B(n x)}-\frac{2 n B^{\prime}(n x)}{(n+\beta) B(n x)}+1\right\} x^{2} \\
& +\left\{\frac{n B^{\prime}(n x)\left[2 A^{\prime}(1)+A(1)\right]}{(n+\beta)^{2} A(1) B(n x)}+\frac{(2 \alpha+1) n B^{\prime}(n x)}{(n+\beta)^{2} B(n x)}\right. \\
& \left.-\frac{2 A^{\prime}(1)}{(n+\beta) A(1)}-\frac{2 \alpha+1}{n+\beta}\right\} x+\frac{A^{\prime \prime}(1)+A^{\prime}(1)}{(n+\beta)^{2} A(1)} \\
+ & \frac{(2 \alpha+1) A^{\prime}(1)}{(n+\beta)^{2} A(1)}+\frac{\alpha^{2}+\alpha+(1 / 3)}{(n+\beta)^{2}} .
\end{aligned}
$$


Theorem 4. Let

$$
\begin{gathered}
E:=\left\{f: x \in[0, \infty), \frac{f(x)}{1+x^{2}} \text { is convergent as } x \rightarrow \infty\right\}, \\
\lim _{y \rightarrow \infty} \frac{B^{\prime}(y)}{B(y)}=1, \quad \lim _{y \rightarrow \infty} \frac{B^{\prime \prime}(y)}{B(y)}=1 .
\end{gathered}
$$

If $f \in C[0, \infty) \cap E$, then

$$
\lim _{n \rightarrow \infty} K_{n}^{(\alpha, \beta)}(f ; x)=f(x)
$$

and the operators $K_{n}^{(\alpha, \beta)}$ converge uniformly in each compact subset of $[0, \infty)$.

Proof. According to Lemma 2, by considering the equality (16), we get

$$
\lim _{n \rightarrow \infty} K_{n}^{(\alpha, \beta)}\left(s^{i} ; x\right)=x^{i}, \quad i=0,1,2 .
$$

This convergence is satisfied uniformly in each compact subset of $[0, \infty)$. Then, the proof follows from the universal Korovkin-type property (vi) of Theorem 4.1.4 in [28].

\section{Rates of Convergence}

In this section, we compute the rates of convergence of the operators $K_{n}^{(\alpha, \beta)}(f)$ to $f$ by means of a classical approach, the second modulus of continuity, and Peetre's $K$-functional.

Let $f \in \widetilde{C}[0, \infty)$. Then for $\delta>0$, the modulus of continuity of $f$ denoted by $w(f ; \delta)$ is defined to be

$$
w(f ; \delta):=\sup _{\substack{x, y \in[0, \infty) \\|x-y| \leq \delta}}|f(x)-f(y)|
$$

where $\widetilde{C}[0, \infty)$ denotes the space of uniformly continuous functions on $[0, \infty)$. Then, for any $\delta>0$ and each $x \in[0, \infty)$, it is well known that one can write

$$
|f(x)-f(y)| \leq w(f ; \delta)\left(\frac{|x-y|}{\delta}+1\right) .
$$

The next result gives the rate of convergence of the sequence $K_{n}^{(\alpha, \beta)}(f)$ to $f$ by means of the modulus of continuity.

Theorem 5. For $f \in \widetilde{C}[0, \infty) \cap E$, one has

$$
\left|K_{n}^{(\alpha, \beta)}(f ; x)-f(x)\right| \leq 2 w\left(f ; \sqrt{\lambda_{n}(x)}\right)
$$

where

$$
\begin{aligned}
\lambda= & \lambda_{n}(x) \\
= & K_{n}^{(\alpha, \beta)}\left((s-x)^{2} ; x\right) \\
= & \left\{\left(\frac{n}{n+\beta}\right)^{2} \frac{B^{\prime \prime}(n x)}{B(n x)}-\frac{2 n B^{\prime}(n x)}{(n+\beta) B(n x)}+1\right\} x^{2} \\
& +\left\{\frac{n B^{\prime}(n x)\left[2 A^{\prime}(1)+A(1)\right]}{(n+\beta)^{2} A(1) B(n x)}+\frac{(2 \alpha+1) n B^{\prime}(n x)}{(n+\beta)^{2} B(n x)}\right. \\
& \left.\quad-\frac{2 A^{\prime}(1)}{(n+\beta) A(1)}-\frac{2 \alpha+1}{n+\beta}\right\} x+\frac{A^{\prime \prime}(1)+A^{\prime}(1)}{(n+\beta)^{2} A(1)} \\
& +\frac{(2 \alpha+1) A^{\prime}(1)}{(n+\beta)^{2} A(1)}+\frac{\alpha^{2}+\alpha+(1 / 3)}{(n+\beta)^{2}} .
\end{aligned}
$$

Proof. Using linearity of the operators $K_{n}^{(\alpha, \beta)}$, (11) and (20), we get

$$
\begin{aligned}
\mid K_{n}^{(\alpha, \beta)} & (f ; x)-f(x) \mid \\
\leq & \frac{n+\beta}{A(1) B(n x)} \sum_{k=0}^{\infty} P_{k}(n x) \\
& \times \int_{(k+\alpha) /(n+\beta)}^{(k+\alpha+1) /(n+\beta)}|f(s)-f(x)| d s \\
\leq & \frac{n+\beta}{A(1) B(n x)} \sum_{k=0}^{\infty} P_{k}(n x) \\
& \times \int_{(k+\alpha) /(n+\beta)}^{(k+\alpha+1) /(n+\beta)}\left(\frac{|s-x|}{\delta}+1\right) w(f ; \delta) d s \\
\leq & \left\{1+\frac{n+\beta}{A(1) B(n x) \delta} \sum_{k=0}^{\infty} P_{k}(n x)\right. \\
& \left.\times \int_{(k+\alpha) /(n+\beta)}^{(k+\alpha+1) /(n+\beta)}|s-x| d s\right\} w(f ; \delta) .
\end{aligned}
$$

According to the Cauchy-Schwarz inequality for integration, we obtain that

$$
\begin{aligned}
& \int_{(k+\alpha) /(n+\beta)}^{(k+\alpha+1) /(n+\beta)}|s-x| d s \\
& \quad \leq \frac{1}{\sqrt{n+\beta}}\left(\int_{(k+\alpha) /(n+\beta)}^{(k+\alpha+1) /(n+\beta)}|s-x|^{2} d s\right)^{1 / 2}
\end{aligned}
$$

from which, it follows that

$$
\begin{aligned}
& \sum_{k=0}^{\infty} P_{k}(n x) \int_{(k+\alpha) /(n+\beta)}^{(k+\alpha+1) /(n+\beta)}|s-x| d s \\
& \quad \leq \frac{1}{\sqrt{n+\beta}} \sum_{k=0}^{\infty} P_{k}(n x)\left(\int_{(k+\alpha) /(n+\beta)}^{(k+\alpha+1) /(n+\beta)}|s-x|^{2} d s\right)^{1 / 2} .
\end{aligned}
$$


By using the Cauchy-Schwarz inequality for summation on the right hand side of (25), we may write

$$
\begin{aligned}
\sum_{k=0}^{\infty} P_{k}(n x) \int_{(k+\alpha) /(n+\beta)}^{(k+\alpha+1) /(n+\beta)}|s-x| d s \\
\quad \leq \frac{\sqrt{A(1) B(n x)}}{\sqrt{n+\beta}}\left(\frac{A(1) B(n x)}{n+\beta} K_{n}^{(\alpha, \beta)}\left((s-x)^{2} ; x\right)\right)^{1 / 2} \\
=\frac{A(1) B(n x)}{n+\beta}\left(K_{n}^{(\alpha, \beta)}\left((s-x)^{2} ; x\right)\right)^{1 / 2} \\
=\frac{A(1) B(n x)}{n+\beta}\left(\lambda_{n}(x)\right)^{1 / 2},
\end{aligned}
$$

where $\lambda_{n}(x)$ is given by (22). Considering this inequality in (23), we find that

$$
\left|K_{n}^{(\alpha, \beta)}(f ; x)-f(x)\right| \leq\left\{1+\frac{1}{\delta} \sqrt{\lambda_{n}(x)}\right\} w(f ; \delta) .
$$

If we set $\delta=\sqrt{\lambda_{n}(x)}$, the proof is completed.

Now, we will study the rates of convergence of the operators $K_{n}^{(\alpha, \beta)}$ to $f$ by means of the second modulus of continuity and Peetre's $K$-functional.

Recall that the second modulus of continuity of $f \in$ $C_{B}[0, \infty)$ is defined by

$$
w_{2}(f ; \delta):=\sup _{0<t \leq \delta}\|f(\cdot+2 t)-2 f(\cdot+t)+f(\cdot)\|_{C_{B}},
$$

where $C_{B}[0, \infty)$ is the class of real valued functions defined on $[0, \infty)$ which are bounded and uniformly continuous with the norm $\|f\|_{C_{B}}=\sup _{x \in[0, \infty)}|f(x)|$.

Peetre's $K$-functional of the function $f \in C_{B}[0, \infty)$ is defined by

$$
K(f ; \delta):=\inf _{g \in C_{B}^{2}[0, \infty)}\left\{\|f-g\|_{C_{B}}+\delta\|g\|_{C_{B}^{2}}\right\}
$$

where

$$
C_{B}^{2}[0, \infty):=\left\{g \in C_{B}[0, \infty): g^{\prime}, g^{\prime \prime} \in C_{B}[0, \infty)\right\}
$$

and the norm $\|g\|_{C_{B}^{2}}:=\|g\|_{C_{B}}+\left\|g^{\prime}\right\|_{C_{B}}+\left\|g^{\prime \prime}\right\|_{C_{B}}$ (see [29]). It is clear that the following inequality:

$$
K(f ; \delta) \leq M\left\{w_{2}(f ; \sqrt{\delta})+\min (1, \delta)\|f\|_{C_{B}}\right\}
$$

holds for all $\delta>0$. The constant $M$ is independent of $f$ and $\delta$.

Theorem 6. Let $f \in C_{B}^{2}[0, \infty)$. If $K_{n}^{(\alpha, \beta)}$ is defined by (9), then one has

$$
\left|K_{n}^{(\alpha, \beta)}(f ; x)-f(x)\right| \leq \zeta\|f\|_{C_{B}^{2}}
$$

where

$$
\begin{aligned}
\zeta= & \zeta_{n}(x) \\
= & \left\{\left(\frac{n}{n+\beta}\right)^{2} \frac{B^{\prime \prime}(n x)}{2 B(n x)}-\frac{n B^{\prime}(n x)}{(n+\beta) B(n x)}+\frac{1}{2}\right\} x^{2} \\
& +\left\{\frac{n B^{\prime}(n x)\left[2 A^{\prime}(1)+(2 \alpha+2) A(1)\right]}{2(n+\beta)^{2} A(1) B(n x)}\right. \\
& +\frac{A^{\prime \prime}(1)+A^{\prime}(1)}{2(n+\beta)^{2} A(1)}+\frac{(2 \alpha+1) A^{\prime}(1)}{2(n+\beta)^{2} A(1)} \\
& +\frac{\alpha^{2}+\alpha+(1 / 3)}{2(n+\beta)^{2}}+\frac{2 A^{\prime}(1)+(2 \alpha+1) A(1)}{2(n+\beta) A(1)} .
\end{aligned}
$$

Proof. We can write from the Taylor expansion of $f$, the linearity of the operators $K_{n}^{(\alpha, \beta)}$, and (11)

$$
\begin{aligned}
K_{n}^{(\alpha, \beta)} & (f ; x)-f(x) \\
= & f^{\prime}(x) K_{n}^{(\alpha, \beta)}(s-x ; x) \\
& +\frac{1}{2} f^{\prime \prime}(\eta) K_{n}^{(\alpha, \beta)}\left((s-x)^{2} ; x\right), \quad \eta \in(x, s) .
\end{aligned}
$$

From Lemma 2, it is obvious that

$$
\begin{aligned}
& K_{n}^{(\alpha, \beta)}(s-x ; x) \\
& \quad=\left\{\frac{n}{n+\beta} \frac{B^{\prime}(n x)}{B(n x)}-1\right\} x+\frac{A^{\prime}(1)}{(n+\beta) A(1)}+\frac{2 \alpha+1}{2(n+\beta)} \geq 0
\end{aligned}
$$

for $s \geq x$. Thus, by considering Lemmas 2 and 3 in (34), one can write

$$
\begin{aligned}
&\left|K_{n}^{(\alpha, \beta)}(f ; x)-f(x)\right| \\
& \leq\left\{\left\{\frac{n}{n+\beta} \frac{B^{\prime}(n x)}{B(n x)}-1\right\} x\right. \\
&\left.+\frac{A^{\prime}(1)}{(n+\beta) A(1)}+\frac{2 \alpha+1}{2(n+\beta)}\right\}\left\|f^{\prime}\right\|_{C_{B}} \\
&+ \frac{1}{2}\left[\left\{\left(\frac{n}{n+\beta}\right)^{2} \frac{B^{\prime \prime}(n x)}{B(n x)}\right.\right. \\
&\left.-\frac{2 n B^{\prime}(n x)}{(n+\beta) B(n x)}+1\right\} x^{2}
\end{aligned}
$$




$$
\begin{aligned}
& +\left\{\frac{n B^{\prime}(n x)\left[2 A^{\prime}(1)+A(1)\right]}{(n+\beta)^{2} A(1) B(n x)}\right. \\
& +\frac{(2 \alpha+1) n B^{\prime}(n x)}{(n+\beta)^{2} B(n x)} \\
& \left.-\frac{2 A^{\prime}(1)}{(n+\beta) A(1)}-\frac{2 \alpha+1}{n+\beta}\right\} x \\
& +\frac{A^{\prime \prime}(1)+A^{\prime}(1)}{(n+\beta)^{2} A(1)}+\frac{(2 \alpha+1) A^{\prime}(1)}{(n+\beta)^{2} A(1)} \\
& \left.+\frac{\alpha^{2}+\alpha+(1 / 3)}{(n+\beta)^{2}}\right]\left\|f^{\prime \prime}\right\|_{C_{B}} \\
& \leq\left[\left\{\left(\frac{n}{n+\beta}\right)^{2} \frac{B^{\prime \prime}(n x)}{2 B(n x)}-\frac{n B^{\prime}(n x)}{(n+\beta) B(n x)}+\frac{1}{2}\right\} x^{2}\right. \\
& +\left\{\frac{n B^{\prime}(n x)\left[2 A^{\prime}(1)+(2 \alpha+2) A(1)\right]}{2(n+\beta)^{2} A(1) B(n x)}\right. \\
& -\frac{2 A^{\prime}(1)+(2 \alpha+1) A(1)}{2(n+\beta) A(1)} \\
& \left.+\frac{n}{n+\beta} \frac{B^{\prime}(n x)}{B(n x)}-1\right\} x \\
& +\frac{A^{\prime \prime}(1)+A^{\prime}(1)}{2(n+\beta)^{2} A(1)}+\frac{(2 \alpha+1) A^{\prime}(1)}{2(n+\beta)^{2} A(1)} \\
& +\frac{\alpha^{2}+\alpha+(1 / 3)}{2(n+\beta)^{2}} \\
& \left.+\frac{2 A^{\prime}(1)+(2 \alpha+1) A(1)}{2(n+\beta) A(1)}\right]\|f\|_{C_{B}^{2}}
\end{aligned}
$$

which completes the proof.

Theorem 7. If $f \in C_{B}[0, \infty)$, then one has

$$
\begin{aligned}
& \left|K_{n}^{(\alpha, \beta)}(f ; x)-f(x)\right| \\
& \quad \leq 2 M\left\{w_{2}(f ; \sqrt{\delta})+\min (1, \delta)\|f\|_{C_{B}}\right\},
\end{aligned}
$$

where

$$
\delta:=\delta_{n}(x)=\frac{1}{2} \zeta_{n}(x)
$$

and $M>0$ is a constant which is independent of the function $f$ and $\delta$. Also, $\zeta_{n}(x)$ is the same as in Theorem 6.
Proof. Suppose that $g \in C_{B}^{2}[0, \infty)$. From Theorem 6, we have

$$
\begin{aligned}
& \left|K_{n}^{(\alpha, \beta)}(f ; x)-f(x)\right| \\
& \quad \leq\left|K_{n}^{(\alpha, \beta)}(f-g ; x)\right|+\left|K_{n}^{(\alpha, \beta)}(g ; x)-g(x)\right| \\
& \quad+|g(x)-f(x)| \\
& \quad \leq 2\|f-g\|_{C_{B}}+\zeta\|g\|_{C_{B}^{2}}=2\left[\|f-g\|_{C_{B}}+\delta\|g\|_{C_{B}^{2}}\right] .
\end{aligned}
$$

Since the left-hand side of inequality (39) does not depend on the function $g \in C_{B}^{2}[0, \infty)$, we get

$$
\left|K_{n}^{(\alpha, \beta)}(f ; x)-f(x)\right| \leq 2 K(f ; \delta),
$$

where $K(f ; \delta)$ is Peetre's $K$-functional defined by (29). By using the relation (31) in (39), the inequality

$$
\left|K_{n}^{(\alpha, \beta)}(f ; x)-f(x)\right| \leq 2 M\left\{w_{2}(f ; \sqrt{\delta})+\min (1, \delta)\|f\|_{C_{B}}\right\}
$$

holds.

Remark 8. In Theorems 5-7, $\lambda_{n}, \zeta_{n}, \delta_{n} \rightarrow 0$ when $n \rightarrow \infty$ under the assumption (16).

\section{Special Cases of the Operators $K_{n}^{(\alpha, \beta)}$ and Further Properties}

Gould-Hopper polynomials $g_{k}^{d+1}(x, h)$ are defined through the identity

$$
g_{k}^{d+1}(x, h)=\sum_{m=0}^{[k /(d+1)]} \frac{k !}{m !(k-(d+1) m) !} h^{m} x^{k-(d+1) m}
$$

and satisfy the generating function

$$
e^{h t^{d+1}} \exp (x t)=\sum_{k=0}^{\infty} g_{k}^{d+1}(x, h) \frac{t^{k}}{k !},
$$

where, as usual, [.] denotes the integer part [30].

The Gould-Hopper polynomials are Brenke-type polynomials for the special case of $A(t)=e^{h t^{d+1}}$ and $B(t)=e^{t}$ in (5). From (2), the operators including the Gould-Hopper polynomials are as follows:

$$
L_{n}^{*}(f ; x):=e^{-n x-h} \sum_{k=0}^{\infty} \frac{g_{k}^{d+1}(n x, h)}{k !} f\left(\frac{k}{n}\right),
$$

where $x \in[0, \infty)$ and $h \geq 0$ (see [11]).

Similarly, the special case $A(t)=e^{h t^{d+1}}$ and $B(t)=e^{t}$ of (9) gives the following Kantorovich-Stancu type operators $K_{n}^{*(\alpha, \beta)}(f ; x)$ including the Gould-Hopper polynomials:

$$
\begin{aligned}
K_{n}^{*(\alpha, \beta)}(f ; x):=(n & +\beta) e^{-n x-h} \sum_{k=0}^{\infty} \frac{g_{k}^{d+1}(n x, h)}{k !} \\
& \times \int_{(k+\alpha) /(n+\beta)}^{(k+\alpha+1) /(n+\beta)} f(t) d t
\end{aligned}
$$

under the assumption $h \geq 0$. 
Remark 9. For $h=0$, we have $g_{k}^{d+1}(n x, 0)=(n x)^{k}$ and the operators given by (45) reduce to the Kantorovich-Stancu type of Szasz-Mirakyan operators given by (10).

Remark 10. For $\alpha=\beta=0$, the operators (45) give the Kantorovich type operators including the Gould-Hopper polynomials given by

$$
K_{n}^{*}(f ; x):=n e^{-n x-h} \sum_{k=0}^{\infty} \frac{g_{k}^{d+1}(n x, h)}{k !} \int_{k / n}^{(k+1) / n} f(t) d t
$$

in $[8]$.

Remark 11. For $h=0$ in Remark 10, we get $g_{k}^{d+1}(n x, 0)=$ $(n x)^{k}$ and then the operators given by (46) reduce to the Szasz-Mirakyan-Kantorovich operators given by (7).

Now, in order to prove a Voronovskaya type theorem for the operators given by (45), let us prove the following lemmas.

Lemma 12. For the operators $K_{n}^{*(\alpha, \beta)}$, one has

$$
\begin{aligned}
& K_{n}^{*(\alpha, \beta)}(1 ; x)=1, \\
& K_{n}^{*(\alpha, \beta)}(s ; x)=\frac{n x}{n+\beta}+\frac{h(d+1)}{n+\beta}+\frac{2 \alpha+1}{2(n+\beta)}, \\
& K_{n}^{*(\alpha, \beta)}\left(s^{2} ; x\right) \\
& =\frac{n^{2} x^{2}}{(n+\beta)^{2}}+\frac{n x}{(n+\beta)^{2}} \\
& \times\{2 h(d+1)+(2 \alpha+2)\}+\frac{1}{(n+\beta)^{2}} \\
& \times\left[h(h+1)(d+1)^{2}+(2 \alpha+1) h(d+1)\right. \\
& \left.+\left(\alpha^{2}+\alpha+\frac{1}{3}\right)\right], \\
& K_{n}^{*(\alpha, \beta)}\left(s^{3} ; x\right) \\
& =\frac{n^{3} x^{3}}{(n+\beta)^{3}}+\frac{3 n^{2} x^{2}}{2(n+\beta)^{3}} \\
& \quad \times\{2 \alpha+3+2(d+1) h\}+\frac{n x}{2(n+\beta)^{3}} \\
& \quad \times\left\{6 h^{2}(d+1)^{2}+6 h(d+1)\right. \\
& \left.\quad \times(3+d+2 \alpha)+12 \alpha+6 \alpha^{2}+7\right\} \\
& +\frac{1}{4(n+\beta)^{3}}\left\{4 h^{3}(d+1)^{3}+6 h^{2}(d+1)^{2}\right. \\
& \times(2 \alpha+2 d+3)+4 \alpha^{3}+6 \alpha^{2} \\
& +4 \alpha+1+2 h(d+1)
\end{aligned}
$$

$$
\begin{gathered}
\times\left[2 d^{2}+d(2 \alpha+7)\right. \\
\left.\left.+12 \alpha+6 \alpha^{2}+7\right]\right\},
\end{gathered}
$$$$
K_{n}^{*(\alpha, \beta)}\left(s^{4} ; x\right)
$$$$
=\frac{n^{4} x^{4}}{(n+\beta)^{4}}+\frac{4 n^{3} x^{3}}{(n+\beta)^{4}}(h(d+1)+\alpha+2)
$$$$
+\frac{3 n^{2} x^{2}}{(n+\beta)^{4}}\left\{2 h^{2}(d+1)^{2}+2 h(d+1)\right.
$$$$
\left.\times(2 \alpha+d+4)+2 \alpha^{2}+6 \alpha+5\right\}
$$$$
+\frac{2 n x}{(n+\beta)^{4}}\left\{2 h^{3}(d+1)^{3}+6 h^{2}(d+1)^{2}(\alpha+d+2)\right.
$$$$
+h(d+1)
$$$$
\times\left(2 d^{2}+10 d+6(3+d) \alpha+6 \alpha^{2}+15\right)
$$$$
+(1+\alpha)(3+2 \alpha(2+\alpha))\}+\frac{1}{5(n+\beta)^{4}}
$$$$
\times\left\{5 h(d+1)^{4}[1+h(7+h(6+h))]\right.
$$$$
+10 h(d+1)^{3}[1+h(3+h)](1+2 \alpha)
$$$$
+10 h(h+1)(d+1)^{2}(1+3 \alpha(1+\alpha))
$$$$
+5 h(d+1)(1+2 \alpha[2+\alpha(3+2 \alpha)])
$$$$
\left.+5 \alpha^{4}+10 \alpha^{3}+10 \alpha^{2}+5 \alpha+1\right\} \text {. }
$$

Proof. The proof follows from the generating function (43) for the Gould-Hopper polynomials.

Lemma 13. For each $x \in[0, \infty)$, one has

$$
\begin{aligned}
K_{n}^{*(\alpha, \beta)}\left((s-x)^{2} ; x\right) & \\
= & \frac{\beta^{2} x^{2}}{(n+\beta)^{2}} \\
+ & {\left[\frac{n}{(n+\beta)^{2}}\{2 h(d+1)+(2 \alpha+2)\}\right.} \\
& \left.-\frac{2 h(d+1)+2 \alpha+1}{n+\beta}\right]+\frac{1}{(n+\beta)^{2}} \\
\times & {\left[h(h+1)(d+1)^{2}\right.} \\
& \left.+(2 \alpha+1) h(d+1)+\left(\alpha^{2}+\alpha+\frac{1}{3}\right)\right], \\
K_{n}^{*(\alpha, \beta)}\left((s-x)^{4} ; x\right)=\frac{\beta^{4} x^{4}}{(n+\beta)^{4}} & \\
- & x^{3}\left\{\frac{2 \beta^{2}(-3 n+(2 h(d+1)+2 \alpha+1) \beta)}{(n+\beta)^{4}}\right\}
\end{aligned}
$$




$$
\begin{aligned}
& +x^{2}\left\{\frac { 1 } { ( n + \beta ) ^ { 4 } } \left[3 n^{2}-2 n \beta(6 h(d+1)+6 \alpha+5)\right.\right. \\
& +2\left\{3 h^{2}(d+1)^{2}+3 h(d+1)\right. \\
& \left.\left.\times(2 \alpha+d+2)+3 \alpha(1+\alpha)+1\} \beta^{2}\right]\right\} \\
& +x\left\{\frac { 2 n } { ( n + \beta ) ^ { 4 } } \left\{2 h^{3}(d+1)^{3}+6 h^{2}(d+1)^{2}\right.\right. \\
& \times(\alpha+d+2)+h(d+1) \\
& \times\left(2 d^{2}+10 d+6(3+d) \alpha\right. \\
& \left.+6 \alpha^{2}+15\right)+(1+\alpha) \\
& \times(3+2 \alpha(2+\alpha))\} \\
& -\frac{1}{(n+\beta)^{3}}\left\{4 h^{3}(d+1)^{3}+6 h^{2}(d+1)^{2}\right. \\
& \times(2 \alpha+2 d+3)+4 \alpha^{3}+6 \alpha^{2} \\
& +4 \alpha+1+2 h(d+1) \\
& \times\left[2 d^{2}+d(2 \alpha+7)+6 \alpha^{2}\right. \\
& +12 \alpha+7]\}\} \\
& +\frac{1}{5(n+\beta)^{4}}\left\{5 h(d+1)^{4}[1+h(7+h(6+h))]\right. \\
& +10 h(d+1)^{3}[1+h(3+h)](2 \alpha+1) \\
& +10(d+1)^{2} h(1+h)(1+3 \alpha(1+\alpha)) \\
& +5 h(d+1)(1+2 \alpha[2+\alpha(3+2 \alpha)]) \\
& \left.+5 \alpha^{4}+10 \alpha^{3}+10 \alpha^{2}+5 \alpha+1\right\} .
\end{aligned}
$$

Proof. From Lemma 12, the proof is obvious.

Theorem 14. Let $f \in C^{2}[0, a]$. Then one has

$$
\begin{aligned}
& \lim _{n \rightarrow \infty}(n+\beta)\left[K_{n}^{*(\alpha, \beta)}(f ; x)-f(x)\right] \\
& \quad=f^{\prime}(x)\left\{\beta x+h(d+1)+\frac{2 \alpha+1}{2}\right\}+\frac{x f^{\prime \prime}(x)}{2 !} .
\end{aligned}
$$

Proof. By Taylor's theorem for $f$, we have

$$
\begin{aligned}
f(s)= & f(x)+(s-x) f^{\prime}(x) \\
& +\frac{(s-x)^{2}}{2 !} f^{\prime \prime}(x)+(s-x)^{2} \eta(s ; x),
\end{aligned}
$$

where $\eta(s ; x) \in C[0, a]$ and $\lim _{s \rightarrow x} \eta(s ; x)=0$. By applying the operator $K_{n}^{*(\alpha, \beta)}$ to the both sides of (50), we have

$$
\begin{gathered}
K_{n}^{*(\alpha, \beta)}(f ; x)=f(x)+f^{\prime}(x) K_{n}^{*(\alpha, \beta)}(s-x ; x) \\
+\frac{f^{\prime \prime}(x)}{2 !} K_{n}^{*(\alpha, \beta)}\left((s-x)^{2} ; x\right) \\
+K_{n}^{*(\alpha, \beta)}\left((s-x)^{2} \eta(s ; x) ; x\right) .
\end{gathered}
$$

According to Lemmas 12 and 13, the equality (51) can be written as follows:

$$
\begin{aligned}
& (n+\beta)\left[K_{n}^{*(\alpha, \beta)}(f ; x)-f(x)\right] \\
& =(n+\beta)\left\{\frac{\beta}{n+\beta} x+\frac{h(d+1)}{n+\beta}+\frac{2 \alpha+1}{2(n+\beta)}\right\} f^{\prime}(x) \\
& +(n+\beta)\left\{x^{2}\left(\frac{\beta}{n+\beta}\right)^{2}\right. \\
& +x\left[\frac{n}{(n+\beta)^{2}}\{2 h(d+1)+(2 \alpha+2)\}\right. \\
& +\frac{1}{(n+\beta)^{2}}\left[h(h+1)(d+1)^{2}+(2 \alpha+1)\right. \\
& \times \frac{f^{\prime \prime}(x)}{2 !}+(n+\beta) K_{n}^{*(\alpha, \beta)}\left((s-x)^{2} \eta(s ; x) ; x\right), \\
& \left.\left.\times h(d+1)+\left(\alpha^{2}+\alpha+\frac{1}{3}\right)\right]\right\}
\end{aligned}
$$

where

$$
\begin{aligned}
& K_{n}^{*(\alpha, \beta)}\left((s-x)^{2} \eta(s ; x) ; x\right) \\
&=(n+\beta) e^{-n x-h} \sum_{k=0}^{\infty} \frac{g_{k}^{d+1}(n x, h)}{k !} \\
& \quad \times \int_{(k+\alpha) /(n+\beta)}^{(k+\alpha+1) /(n+\beta)}(s-x)^{2} \eta(s ; x) d s .
\end{aligned}
$$

By applying Cauchy-Schwarz inequality, we can write

$$
\begin{aligned}
(n+ & \beta) K_{n}^{*(\alpha, \beta)}\left((s-x)^{2} \eta(s ; x) ; x\right) \\
\leq & (n+\beta)^{2} e^{-n x-h} \sum_{k=0}^{\infty} \frac{g_{k}^{d+1}(n x, h)}{k !} \\
& \times\left(\int_{(k+\alpha) /(n+\beta)}^{(k+\alpha+1) /(n+\beta)}(s-x)^{4} d s\right)^{1 / 2} \\
& \times\left(\int_{(k+\alpha) /(n+\beta)}^{(k+\alpha+1) /(n+\beta)} \eta^{2}(s ; x) d s\right)^{1 / 2} .
\end{aligned}
$$


If we consider Cauchy-Schwarz inequality again on the righthand side of inequality above, then we arrive at

$$
\begin{aligned}
(n+\beta) & K_{n}^{*(\alpha, \beta)}\left((s-x)^{2} \eta(s ; x) ; x\right) \\
\leq & \left((n+\beta)^{3} e^{-n x-h} \sum_{k=0}^{\infty} \frac{g_{k}^{d+1}(n x, h)}{k !}\right. \\
& \left.\times \int_{(k+\alpha) /(n+\beta)}^{(k+\alpha+1) /(n+\beta)}(s-x)^{4} d s\right)^{1 / 2} \\
& \times\left((n+\beta) e^{-n x-h} \sum_{k=0}^{\infty} \frac{g_{k}^{d+1}(n x, h)}{k !}\right. \\
& \left.\times \int_{(k+\alpha) /(n+\beta)}^{(k+\alpha+1) /(n+\beta)} \eta^{2}(s ; x) d s\right)^{1 / 2} \\
= & \sqrt{(n+\beta)^{2} K_{n}^{*(\alpha, \beta)}\left((s-x)^{4} ; x\right)} \sqrt{K_{n}^{*(\alpha, \beta)}\left(\eta^{2}(s ; x) ; x\right) .}
\end{aligned}
$$

From Lemma 13, we have

$$
\lim _{n \rightarrow \infty}(n+\beta)^{2} K_{n}^{*(\alpha, \beta)}\left((s-x)^{4} ; x\right)=3 x^{2} .
$$

On the other hand, since $\eta(s ; x) \in C[0, a]$ and $\lim _{s \rightarrow x} \eta(s$; $x)=0$, then it follows from Theorem 4 that

$$
\lim _{n \rightarrow \infty} K_{n}^{*(\alpha, \beta)}\left(\eta^{2}(s ; x) ; x\right)=\eta^{2}(x ; x)=0 .
$$

Therefore, we conclude from (55), (56), and (57) that

$$
\lim _{n \rightarrow \infty}(n+\beta) K_{n}^{*(\alpha, \beta)}\left((s-x)^{2} \eta(s ; x) ; x\right)=0
$$

and then, by taking limit as $n \rightarrow \infty$ in (52) and using (58), we find

$$
\begin{aligned}
& \lim _{n \rightarrow \infty}\left[K_{n}^{*(\alpha, \beta)}(f ; x)-f(x)\right] \\
& \quad=f^{\prime}(x)\left\{\beta x+h(d+1)+\frac{2 \alpha+1}{2}\right\}+\frac{x f^{\prime \prime}(x)}{2 !}
\end{aligned}
$$

which completes the proof.

Remark 15. For $\alpha=\beta=0$, Theorem 14 represents the Voronovskaya type theorem for the operators given by (46) (see [8]).

Remark 16. For $h=0$, it yields a Voronovskaya type theorem for the Kantorovich-Stancu type of Szasz-Mirakyan operators given by (10).

Remark 17. Getting $\alpha=\beta=h=0$ in Theorem 14 gives the Voronovskaya type result for the Szasz-MirakyanKantorovich operators given by (7).

\section{References}

[1] O. Szasz, "Generalization of S. Bernstein's polynomials to the infinite interval," Journal of Research of the National Bureau of Standards, vol. 45, pp. 239-245, 1950.
[2] A. Altın, O. Doğru, and F. Taşdelen, "The generalization of Meyer-König and Zeller operators by generating functions," Journal of Mathematical Analysis and Applications, vol. 312, no. 1, pp. 181-194, 2005.

[3] O. Dogru, M. A. Özarslan, and F. Taşdelen, "On positive operators involving a certain class of generating functions," Studia Scientiarum Mathematicarum Hungarica, vol. 41, no. 4, pp. 415429, 2004.

[4] G. İçöz, F. Taşdelen Yeşildal, and O. Doğru, "Kantrorovich process of linear positive operators via biorthogonal polynomials," Journal of Inequalities and Special Functions, vol. 3, pp. 77-84, 2012.

[5] G. İçöz, F. T. Yeşildal, and S. Varma, "On linear positive operators involving biorthogonal polynomial," Ars Combinatoria, vol. 105, pp. 319-331, 2012.

[6] M. A. Özarslan, " $q$-Laguerre type linear positive operators," Studia Scientiarum Mathematicarum Hungarica, vol. 44, no. 1, pp. 65-80, 2007.

[7] M. A. Özarslan and O. Duman, "Approximation properties of Poisson integrals for orthogonal expansions," Taiwanese Journal of Mathematics, vol. 12, no. 5, pp. 1147-1163, 2008.

[8] F. Taşdelen, R. Aktaş, and A. Altın, "A Kantorovich type of szasz operators including brenke-type polynomials," Abstract and Applied Analysis, vol. 2012, Article ID 867203, 13 pages, 2012.

[9] F. Taşdelen and A. Erençin, "The generalization of bivariate $\mathrm{MKZ}$ operators by multiple generating functions," Journal of Mathematical Analysis and Applications, vol. 331, no. 1, pp. 727735, 2007.

[10] S. Varma, G. İçöz, and S. Sucu, "On some extensions of Szasz operators including Boas-Buck-type polynomials," Abstract and Applied Analysis, vol. 2012, Article ID 680340, 15 pages, 2012.

[11] S. Varma, S. Sucu, and G. İçöz, "Generalization of Szasz operators involving Brenke type polynomials," Computers \& Mathematics with Applications, vol. 64, no. 2, pp. 121-127, 2012.

[12] S. Varma and F. Taşdelen, "Szasz type operators involving Charlier polynomials," Mathematical and Computer Modelling, vol. 56, no. 5-6, pp. 118-122, 2012.

[13] S. Varma and F. Taşdelen, "On a generalization of SzaszDurrmeyer operators with some orthogonal polynomials," Studia Universitatis Babeş-Bolyai, vol. 58, no. 2, pp. 225-232, 2013.

[14] A. Jakimovski and D. Leviatan, "Generalized Szasz operators for the approximation in the infinite interval," Mathematica, vol. 11, no. 34, pp. 97-103, 1969.

[15] M. E.-H. Ismail, “On a generalization of Szasz operators," Mathematica, vol. 39, no. 2, pp. 259-267, 1974.

[16] T. S. Chihara, An Introduction to Orthogonal Polynomials, vol. 13, Gordon and Breach Science Publishers, New York, NY, USA, 1978.

[17] P. L. Butzer, "On the extensions of Bernstein polynomials to the infinite interval," Proceedings of the American Mathematical Society, vol. 5, pp. 547-553, 1954.

[18] O. Duman, M. A. Özarslan, and B. D. Vecchia, "Modified SzaszMirakjan-Kantorovich operators preserving linear functions," Turkish Journal of Mathematics, vol. 33, no. 2, pp. 151-158, 2009.

[19] D. Miclaus, “The Voronovskaja type theorem for the SzászMirakjan-Kantorovich operators," Journal of Science and Arts, vol. 2, no. 13, pp. 257-260, 2010.

[20] G. Nowak and A. Sikorska-Nowak, "Some approximation properties of modified Szasz-Mirakyan-Kantorovich operators," Revue d'Analyse Numérique et de Théorie de l'Approximation, vol. 38, no. 1, pp. 73-82, 2009. 
[21] V. Totik, "Approximation by Szasz-Mirakjan-Kantorovich operators in $L^{p}(p>1)$," Analysis Mathematica, vol. 9, no. 2, pp. 147$167,1983$.

[22] G. Başcanbaz-Tunca and A. Erençin, "A Voronovskaya type theorem for q-Szasz-Mirakyan-Kantorovich operators," Revue d'Analyse Numérique et de Théorie de l'Approximation, vol. 40, no. 1, pp. 14-23, 2011.

[23] Z. Walczak, "On approximation by modified Szasz-Mirakyan operators," Glasnik Matematički, vol. 37, no. 2, pp. 303-319, 2002.

[24] Ç. Atakut and İ. Büyükyazıcı, "Stancu type generalization of the Favard-Szàsz operators," Applied Mathematics Letters, vol. 23, no. 12, pp. 1479-1482, 2010.

[25] D. Bărbosu, "Kantorovich-Stancu type operators," Journal of Inequalities in Pure and Applied Mathematics, vol. 5, no. 3, article 53, 2004.

[26] İ. Büyükyazıcı, "Approximation by Stancu-Chlodowsky polynomials," Computers \& Mathematics with Applications, vol. 59, no. 1, pp. 274-282, 2010.

[27] A. Erençin, G. Başcanbaz-Tunca, and F. Taşdelen, "Kantorovich type q-Bernstein-Stancu operators," Studia Universitatis BabeşBolyai Mathematica, vol. 57, no. 1, pp. 89-105, 2012.

[28] F. Altomare and M. Campiti, Korovkin-Type Approximation Theory and Its Applications, vol. 17 of de Gruyter Studies in Mathematics, Walter de Gruyter, Berlin, Germany, 1994.

[29] Z. Ditzian and V. Totik, Moduli of Smoothness, vol. 9 of Springer Series in Computational Mathematics, Springer, New York, NY, USA, 1987.

[30] H. W. Gould and A. T. Hopper, "Operational formulas connected with two generalizations of Hermite polynomials," Duke Mathematical Journal, vol. 29, pp. 51-63, 1962. 


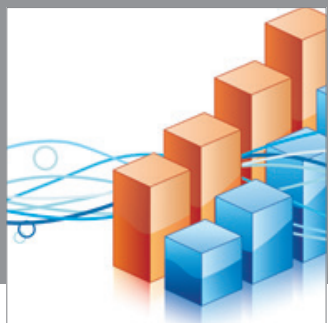

Advances in

Operations Research

mansans

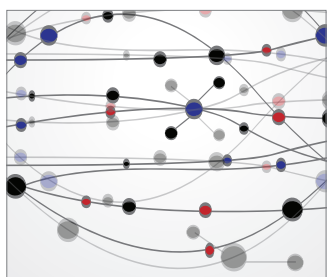

The Scientific World Journal
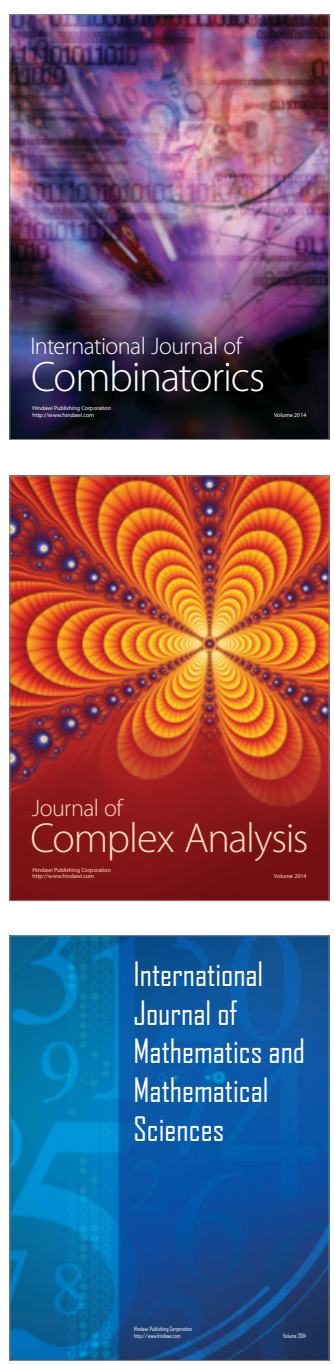
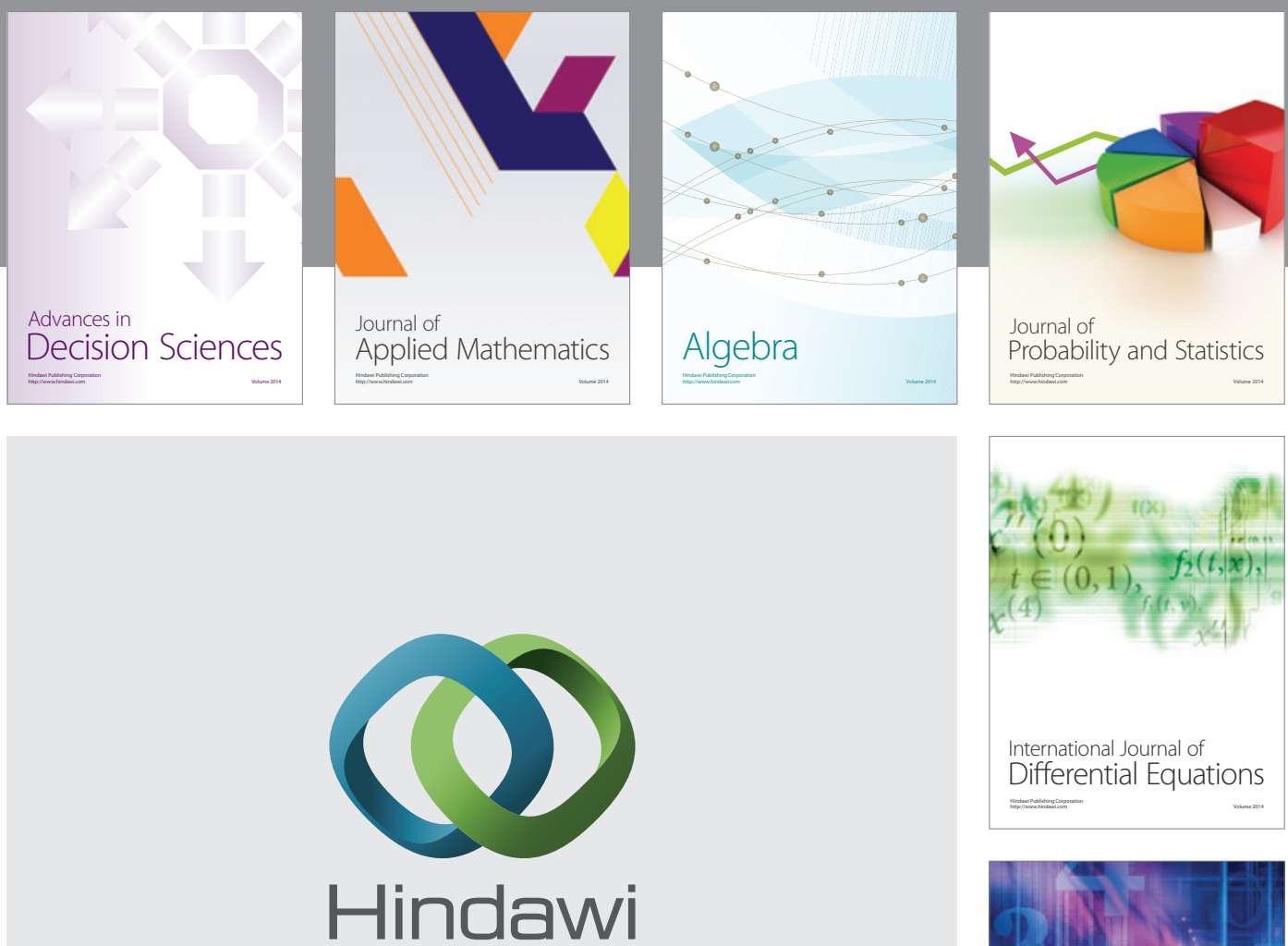

Submit your manuscripts at http://www.hindawi.com
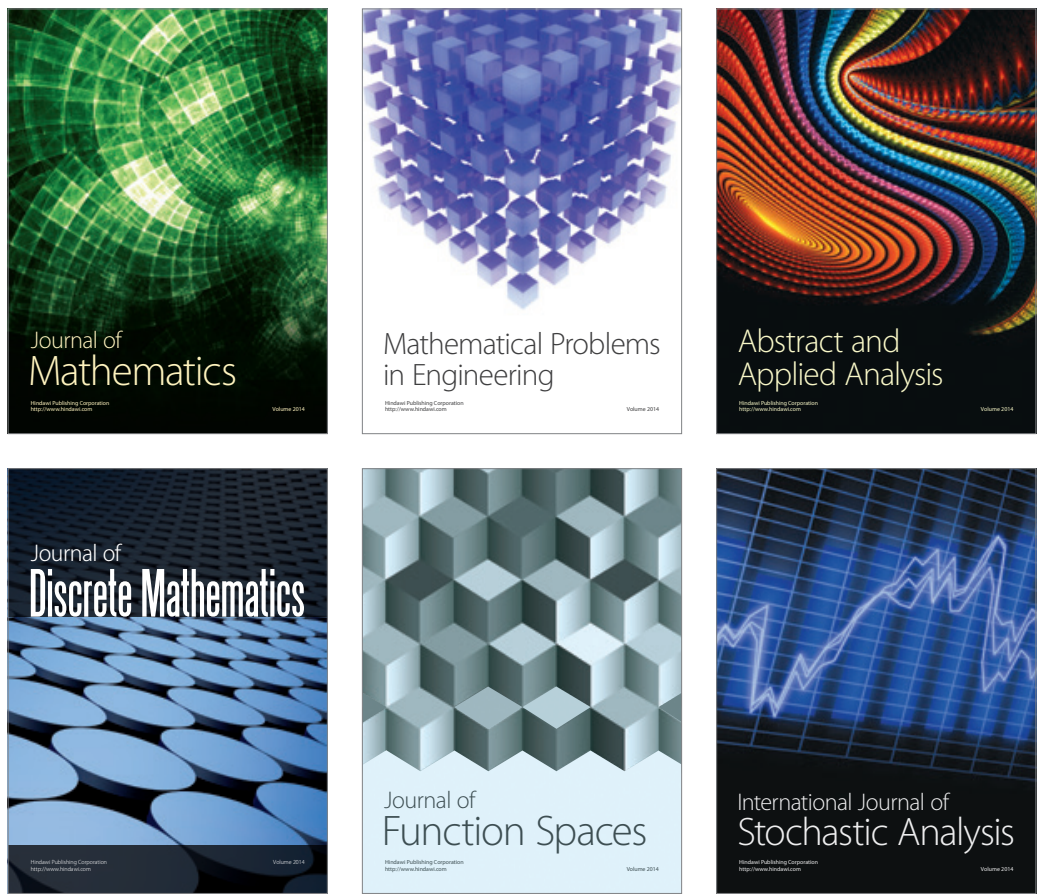

Journal of

Function Spaces

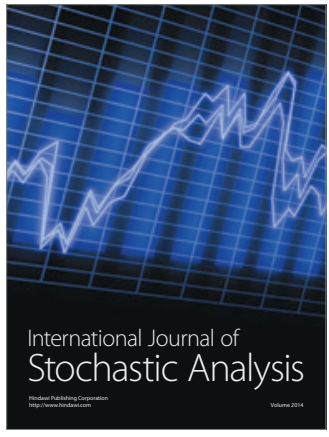

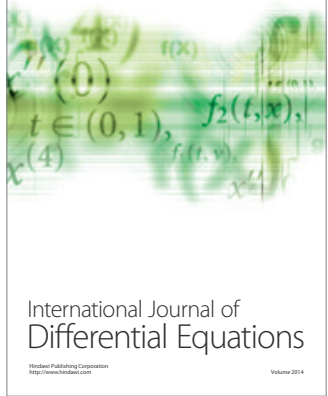
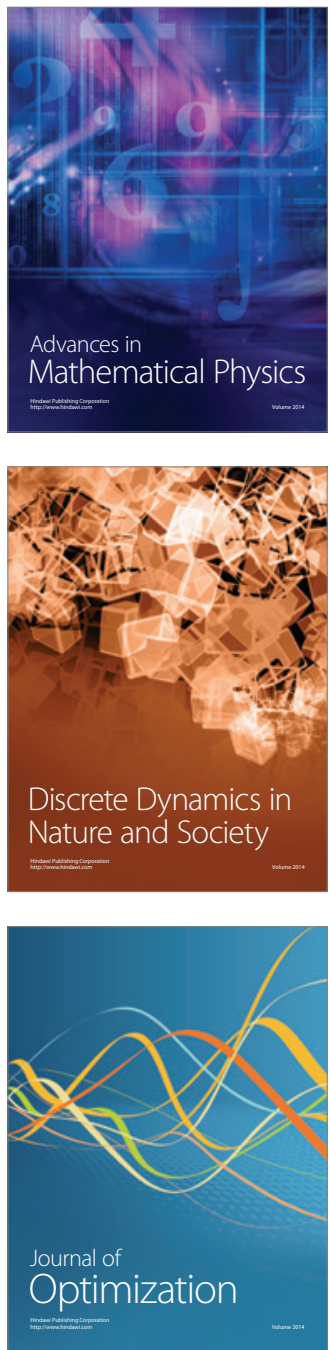